\title{
Azole-resistant Aspergillus fumigatus due to TR46/ Y121F/T289A mutation emerging in Belgium, July 2012
}

E Vermeulen ${ }^{1}$, J Maertens ${ }^{2}$, H Schoemans², K Lagrou (katrien.lagrou@uzleuven.be) ${ }^{1,3}$

1. Catholic University of Leuven, Department of Microbiology and Immunology, Leuven, Belgium

2. University Hospitals Leuven, Department of Hematology, Leuven, Belgium

3. University Hospitals Leuven, Department of Laboratory Medicine, Leuven, Belgium

Citation style for this article:

Vermeulen E, Maertens J, Schoemans H, Lagrou K. Azole-resistant Aspergillus fumigatus due to TR46/Y121F/T289A mutation emerging in Belgium, July 2012. Euro Surveill. 2012;17(48):pii=20326. Available online: http://www.eurosurveillance.org/ViewArticle.aspx?Articleld=20326

Article submitted on 16 November 2012 / published on 29 November 2012

A new azole resistance mechanism in Aspergillus fumigatus consisting of a TR46/Y121F/T289A alteration in the cyp51A gene was recently described in the Netherlands. Strains containing these mutations are associated with invasive infection and therapy failure. This communication describes the first case of fatal invasive aspergillosis caused by TR46/Y121F/T289A outside the Netherlands, in the neighboring country of Belgium, suggesting geographical spread. TR46/ $\mathrm{Y}_{121 \mathrm{~F}} / \mathrm{T}_{28}$ 9A leads to a recognisable phenotypic susceptibility pattern which should trigger cyp51A genotyping to monitor further spread.

\section{Case report}

A 57-year-old male, diagnosed with stage IIIA multiple myeloma (IgG kappa) in 2009, received a fully matched, unrelated haematopoietic stem cell transplantation following reduced-intensity conditioning (fludarabinemelphalan-ATG) in May 2012. Prior treatment regimens included multiple lines of chemotherapy, autologous transplantation, proteasome inhibitors (bortezomib), immunomodulatory agents (lenalidomide) and highdose corticosteroids. At the time of transplantation, the patient had achieved a very good partial response ( $\$ 10 \%$ residual monoclonal paraprotein). The posttransplantation course was complicated by grade III hyperacute graft-versus-host disease (GVHD), involving mainly the skin and the gastro-intestinal tract. Methylprednisolone was started at $2 \mathrm{mg} / \mathrm{kg}$ and slowly tapered over the following weeks. However, high-dose corticotherapy needed to be re-installed in June 2012 because of a relapse of grade III acute GVHD. The patient was receiving fluconazole $400 \mathrm{mg}$ daily since May 2012 as prophylaxis, but was never exposed to mold-active azoles.

One month later, in July 2012, the patient presented with dyspnea, pleuritic-type chest pain and fever, up to $39.9^{\circ} \mathrm{C}$. Thoracic computed tomography (CT)-scan imaging showed multiple ill-defined lesions surrounded by ground glass opacities, suggestive of angio-invasive pulmonary mold infection. Serum galactomannan testing was repeatedly positive (maximum index 5.2; norm
(0.5). Galactomannan detection in broncho-alveolar lavage (BAL) fluid tested positive as well (index 5.8), and Aspergillus fumigatus was cultured from BAL fluid. A diagnosis of probable pulmonary invasive aspergillosis (IA) was made following revised European Organization for Research and Treatment of Cancer/Mycoses Study Group (EORTC/MSG) criteria [1]; the patient agreed to participate in a double-blinded phase III clinical trial comparing two azoles with anti-Aspergillus activity. However, serum galactomannan levels did not decrease while he received azole therapy and the clinical condition of the patient deteriorated rapidly.

Meanwhile, the isolate was tested for azole susceptibility, following Clinical and Laboratory Standards Institute (CSLI) protocols and showed an azole-resistant phenotype, with high-grade resistance to voriconazole (minimal inhibitory concentration (MIC) >16 mg/L) and less pronounced resistance to itraconazole (MIC $=4 \mathrm{mg} / \mathrm{L}$ ) and posaconazole $(\mathrm{MIC}=1 \mathrm{mg} / \mathrm{L})$. Formal clinical breakpoints have not been established by CLSI for Aspergillus susceptibility testing. Based on the epidemiological cut-off values (wild-type distributions), resistance to itraconazole is defined as an MIC >1 $\mathrm{mg} / \mathrm{L}$, to voriconazole as $>1 \mathrm{mg} / \mathrm{L}$ and to posaconazole as $>0.5 \mathrm{mg} / \mathrm{L}$ [2]. An excellent essential agreement (EA) between CLSI and EUCAST methods was described in susceptibility testing of $A$. fumigatus to these azoles [3] and EUCAST already established breakpoints for itraconazole and posaconazole (itraconazole $\leq 1 \mathrm{mg} / \mathrm{L}$ is considered susceptible and $>2 \mathrm{mg} / \mathrm{L}$ resistant; posaconazole $\leq 0.12$ is considered susceptible and $>0.25$ resistant) [4]. A recent report, using an in vitro dynamic model of pulmonary IA that enabled simulation of human voriconazole pharmacokinetics, proposed CLSI breakpoints for voriconazole as $\leq 0.5 \mathrm{mg} / \mathrm{L}$ for susceptible and >1 $\mathrm{mg} / \mathrm{L}$ for resistant [5].

Given this new finding of azole resistance and the rapid clinical decline, the investigators decided to withdraw the patient from the clinical study. Nine days after the start of azole therapy, liposomal amphotericin B was started at a dose of $3 \mathrm{mg} / \mathrm{kg}$. Nevertheless, the patient 
developed widespread IA with eye and brain involvement. A brain magnetic resonance imaging (MRI) scan taken 15 days after the initial diagnosis of invasive aspergillosis showed multiple nodular non-contrastenhancing lesions suggestive of cerebral aspergillosis; this was confirmed by positive galactomannan testing in cerebrospinal fluid (index 4.8). The patient died 19 days after his first presentation with dyspnea. Azole resistance in the strain affecting the patient was shown to be due to cyp51A mutation TR46/Y121F/T289A.

\section{Characterisation of the Aspergillus}

\section{isolate derived from the patient}

The Aspergillus isolate, cultured from BAL fluid, was identified as Aspergillus fumigatus complex based on microscopic and macroscopic characteristics. This identification was confirmed to the species level with beta-tubulin sequencing, as described previously [6]. The isolate was tested for susceptibility with broth microdilution following the CLSI M38-A2 protocol [7]. Genotypic identification of the resistance mechanism was performed by sequencing of the cyp $51 \mathrm{~A}$ gene, as described previously [8].

\section{Discussion and conclusion}

Invasive aspergillosis is an important infectious complication in haematologic patients [9], but also in other groups of immunocompromised and intensive care patients [10]. Triazoles are the mainstay of therapy, with voriconazole the first-line therapy for IA [11]. However, reports of azole resistance have emerged, not only after long-term azole exposure [12], but also after short-term exposure and in azole-naïve patients [13]. In the Netherlands, over $90 \%$ of the resistant clinical strains were attributable to the same resistance mechanism [13]. Therefore, an environmental route of resistance development is assumed and this is suspected to be related to the selective pressure of azole fungicides in the environment [14]. This predominant resistance mechanism is mediated by a tandem repeat of 34 bases (TR34) in the promoter region of the cyp51a gene and a substitution at position $98\left(\mathrm{TR}_{34} / \mathrm{L} 98 \mathrm{H}\right)$, which encodes a residue of the azole target, sterol 14-alphademethylase. This resistance mechanism, conferring pan-azole resistance, has to date spread across Europe as well as to China and India $[8,12,13,15-17]$. Because of the widespread and abundant consumption of azole fungicides in agriculture, there is a risk that other resistance mechanisms might emerge in the environment as well [14]. Recently, in October 2011, a new resistance mechanism due to a 46 base tandem repeat (TR46) in the promoter of the cyp51a gene and two point mutations (TR46/Y121F/T289A) was described in persons with IA who failed therapy in the Netherlands [18].

To our knowledge, the case described in this report is the first case of azole resistance in A. fumigatus due to $T R 46 / Y_{121} \mathrm{~F} / \mathrm{T}_{2} 89 \mathrm{~A}$ outside the Netherlands. The patient lived about 60 kilometres from the Dutch border and did not have any recent travel history to the Netherlands. TR46/Y121F/T289A-bearing strains have also been found throughout the environment in Belgium, among azole-resistant $A$. fumigatus isolates cultured from outdoor air sampling, which was performed from June to September 2012 (data not shown). This case confirms the geographic spread of this new resistance mechanism, possibly following the same path as $T R 34 / \mathrm{L} 98 \mathrm{H}$, which now causes therapy-resistant infections across Europe and even outside Europe $[8,12,13,15-17]$. The phenotype of the TR46/Y121F/ T289A strains consists of a very high MIC to voriconazole ( $>16 \mathrm{mg} / \mathrm{L})$, and an itraconazole MIC which is often multiple dilutions lower. In contrast, in $\mathrm{TR}_{34} / \mathrm{L} 98 \mathrm{H}$ mutated strains, itraconazole MICs are typically higher than voriconazole MICs. This finding (MIC voriconazole $>16 \mathrm{mg} / \mathrm{L}$ and voriconazole MIC $\geq \mathrm{MIC}$ itraconazole) should raise awareness of this new TR46/Y121F/T289A resistance mechanism in other centres and countries.

Susceptibility testing should not delay initiation of therapy. Culture has a low sensitivity and takes about $48 \mathrm{~h}$ to become positive; susceptibility testing takes at least another $48 \mathrm{~h}$. Resistance is therefore often a late finding in the management of the individual patient. Molecular techniques are a promising tool to rapidly provide information about resistance genotype, but clinicians should be aware that they are often designed to detect known resistance mechanisms and can therefore miss new mutations. On the other hand, not all mutations necessarily lead to a resistant phenotype [19]. Surveillance programs are crucial to monitor the local epidemiology of azole resistance, to correctly assess the risk of resistance associated with current treatment strategies. Susceptibility testing in individual patients with invasive aspergillosis should not be delayed until treatment failure because of the life-threatening character of this disease which is illustrated by this case.

\section{Acknowledgments}

EV receives a grant from Research Foundation Flandres (Fonds Wetenschappelijk Onderzoek Vlaanderen). An epidemiological study including typing and susceptibility testing of clinically relevant Aspergillus isolates has been approved by the local Ethics Committee University Hospitals Leuven, with reference $\mathrm{S}_{53024}$.

\section{Conflicts of interest}

Potential conflicts of interest are listed as follows. JM has served as consultant to Schering-Plough, Gilead Sciences, Merck, Sharp \& Dohme, Pfizer Inc., Bio-Rad, Fujisawa healthcare, Inc., Astellas, Nextar and Zeneus (Cephalon). JM has received research funding from Bio-Rad, Merck, Sharp \& Dohme, and Pfizer Inc. JM has been on the speaker's bureau for Schering-Plough, Gilead Sciences, Merck, Sharp \& Dohme, Pfizer Inc., Bio-Rad, Fujisawa healthcare, Inc, Astellas and Zeneus (Cephalon). KL has received research grants from Gilead Sciences, Pfizer Inc. and Merck, Sharp $\&$ Dohme and served on the speakers' bureau of Pfizer Inc. and Merck, Sharp \& Dohme. HS has served as consultant to Bristol Meyer Squibb. 


\section{References}

1. De Pauw B, Walsh TJ, Donnelly JP, Stevens DA, Edwards JE, Calandra T, et al. Revised definitions of invasive fungal disease from the European Organization for Research and Treatment of Cancer/Invasive Fungal Infections Cooperative Group and the National Institute of Allergy and Infectious Diseases Mycoses Study Group (EORTC/MSG) Consensus Group. Clin Infect Dis. 2008;46(12):1813-21.

2. Espinel-Ingroff A, Diekema DJ, Fothergill A, Johnson E, Pelaez T, Pfaller MA, et al. Wild-type MIC distributions and epidemiological cutoff values for the triazoles and six Aspergillus spp. for the CLSI broth microdilution method (M38-A2 document). J Clin Microbiol. 2010;48(9):3251-7.

3. Pfaller M, Boyken L, Hollis R, Kroeger J, Messer S, Tendolkar $\mathrm{S}$, et al. Comparison of the broth microdilution methods of the European Committee on Antimicrobial Susceptibility Testing and the Clinical and Laboratory Standards Institute for testing itraconazole, posaconazole, and voriconazole against Aspergillus isolates. J Clin Microbiol. 2011;49(3):1110-2.

4. European Committee on Antimicrobial Susceptibility Testing (EUCAST). Antifungal Agents - breakpoint tables for interpretation of MICS. 2012; version 4.1. Växjö: EUCAST. 14 Mar 2012. Available from: http://www.eucast. org/fileadmin/src/media/PDFs/EUCAST_files/AFST/ Antifungal_breakpoints_v_4.1.pdf

5. Jeans AR, Howard SI, Al-Nakeeb Z, Goodwin J, Gregson L, Majithiya JB, et al. Pharmacodynamics of voriconazole in a dynamic in vitro model of invasive pulmonary aspergillosis: implications for in vitro susceptibility breakpoints. J Infect Dis. 2012;206(3):442-52.

6. Snelders E, Karawajczyk A, Schaftenaar G, Verweij P, Melchers WJG. Azole Resistance profile of amino acid changes in Aspergillus fumigatus CYP $51 \mathrm{~A}$ based on protein homology modeling. Antimicrob Agents Chemother. 2010;54(6):2425-30.

7. Clinical and Laboratory Standards Institute (CLSI). Reference method for broth dilution antifungal susceptibility testing of filamentous fungi: approved standard. 2nd ed. M38-A2 Vol. 28 No. 16. Wayne (Pensylvania): CLSI; 2008.

8. Alanio A, Sitterlé E, Liance M, Farrugia C, Foulet F, Botterel $\mathrm{F}$, et al. Low prevalence of resistance to azoles in Aspergillus fumigatus in a French cohort of patients treated for haematological malignancies. J Antimicrob Chemother. 2011;66(2):371-4.

9. Kuderer NM, Dale DC, Crawford J, Cosler LE, Lyman GH. Mortality, morbidity, and cost associated with febrile neutropenia in adult cancer patients. Cancer. 2006;106(10):2258-66.

10. Meersseman W, Vandecasteele SJ, Wilmer A, Verbeken E, Peetermans WE, Van Wijngaerden E. Invasive aspergillosis in critically ill patients without malignancy. Am J Respir Crit Care Med. 2004;170(6):621-5.

11. Walsh TJ, Anaissie EJ, Denning DW, Herbrecht R, Kontoyiannis DP, Marr KA, et al. Treatment of aspergillosis: clinical practice guidelines of the Infectious Diseases Society of America. Clin Infect Dis. 2008;46(3):327-60.

12. Howard SJ, Cerar D, Anderson MJ, Albarrag A, Fisher MC, Pasqualotto AC, et al. Frequency and evolution of Azole resistance in Aspergillus fumigatus associated with treatment failure. Emerg Infect Dis. 2009;15(7):1068-76.

13. Snelders E, van der Lee HA, Kuijpers J, Rijs AJ, Varga J, Samson $\mathrm{RA}$, et al. Emergence of azole resistance in Aspergillus fumigatus and spread of a single resistance mechanism. PLoS Med. 2008;5(11)e219:1629-37.

14. Verweij PE, Snelders E, Kema GH, Mellado E, Melchers WJ. Azole resistance in Aspergillus fumigatus: a sideeffect of environmental fungicide use? Lancet Infect Dis. 2009;9(12):789-95

15. Van der Linden JWM, Arendrup MC, Verweij PE, SCARE network. Prospective international surveillance of azole resistance in Aspergillus fumigatus: SCARE-Network. In: 51st Interscience Conference on Antimicrobial Agents and Chemotherapy (ICAAC); 2011 Sep 17-20; Chicago, IL. Abstract M-490.

16. Lockhart SR, Frade JP, Etienne KA, Pfaller MA, Diekema DJ, Balajee SA. Azole resistance in Aspergillus fumigatus isolates from the ARTEMIS global surveillance study is primarily due to the TR/L98H mutation in the cyp51A gene. Antimicrob Agents Chemother. 2011;55(9):4465-8.

17. Chowdhary A, Kathuria S, Randhawa HS, Gaur SN, Klaassen $\mathrm{CH}$, Meis JF. Isolation of multiple-triazole-resistant Aspergillus fumigatus strains carrying the TR/L98H mutations in the cyp51A gene in India. J Antimicrob Chemother. 2012;67(2):362-6.

18. Camps SMT, van der Linden JWM, Melchers WJG, Verweij PE. A new resistance mechanism emerging in clinical Aspergillus fumigatus isolates in The Netherlands. In: 5 th TIMM, Valencia, Spain, 2011, Abstract 03.2.

19. Howard SJ, Arendrup MC. Acquired antifungal drug resistance in Aspergillus fumigatus: epidemiology and detection. Med Mycol. 2011;49 Suppl 1:S90-5. 